\title{
THE EFFECT OF SLIP VELOCITY ON UNSTEADY PERISTALSIS MHD BLOOD FLOW THROUGH A CONSTRICTED ARTERY EXPERIENCING BODY ACCELERATION
}

\author{
J. NANDAL and S. KUMARI* \\ Department of Mathematics, M.D. University \\ Rohtak-124001, Haryana, INDIA \\ E-mails: shooda.mdu@gmail.com; jagdishnandal@gmail.com \\ R. RATHEE \\ A.I.J.H.M. College \\ Rohtak -124001, Haryana, INDIA \\ E-mail: anjalisidharth@gmail.com
}

\begin{abstract}
In this analysis, we present a theoretical study to examine the combined effect of both slip velocity and periodic body acceleration on an unsteady generalized non-Newtonian blood flow through a stenosed artery with permeable wall. A constant transverse magnetic field is applied on the peristaltic flow of blood, treating it as an elastico-viscous, electrically conducting and incompressible fluid. Appropriate transformation methods are adopted to solve the unsteady non-Newtonian axially symmetric momentum equation in the cylindrical polar coordinate system with suitably prescribed conditions. To validate the applicability of the proposed analysis, analytical expressions for the axial velocity, fluid acceleration, wall shear stress and volumetric flow rate are computed and for having an adequate insight to blood flow behavior through a stenosed artery, graphs have been plotted with varying values of flow variables, to analyse the influence of the axial velocity, wall shear stress and volumetric flow rate of streaming blood.
\end{abstract}

Key words: elastico-viscous, slip velocity, selective permeability, peristalsis, stenosed artery.

Chinese Library Classification: 310

Mathematics Subject Classification (2010): 76Zxx, 76Wxx

\section{Introduction}

Today rheology of streaming blood in human arteries is a very significant aspect of research due to feasible medical and engineering applications and recently has attracted attention of researchers since the malfunctioning of the cardiovascular system is the most world wide-spread disease in humans. Systematic blood circulation all over the body through blood vessels is the base of our life. Peristaltic pumping by heart is to supply nutrients through oxygen rich blood to each and every cell of a human body under a sufficient pressure induced by a progressive wave of regular contraction and expansion of the heart and then blood or (bodily fluid) returns along with cellular waste. But blood peristalsis is not always so regular since transport of blood depends on its hydrodynamic behaviour and mechanical properties of blood vessel walls. When a normal blood flow in an artery is disturbed, it may be the cause of development of many cardiovascular diseases, especially: hypertension, hypotension, heart attack, stroke, etc. and particularly atherosclerosis (athero means gruel or paste and sclerosis means hardness), medically called stenosis. It is a common disease developed by intimal thickening of artery's lumen, due to deposition of arteriosclerotic plaque or other types of abnormal tissues along the wall of blood vessel, narrowing the artery through which blood has to pass with relatively high pressure. Also, eventually necrosis may occur, when blood supply to a tissue is blocked or reduced. Therefore, the analysis of blood flow in stenosed arteries is of great importance.

\footnotetext{
* To whom correspondence should be addressed
} 
To examine the flow characteristics of blood through stenosed vessel, Beaver and Joseph [1] employed boundary conditions at a naturally permeable vessel wall. It would help in understanding why noslip condition at vessel wall should be replaced by slip velocity. Oka and Murata [2] presented hydrodynamical theory for flow of blood through a blood vessel with permeable wall and investigated the exchange of fluid as a steady slow motion across the permeable wall upon the motion of the fluid within rigid circular tube. Saffman [3] discussed boundary conditions for flow of fluid at the surface of a porous medium. Popel et al. [4] investigated a continuum approach to blood flow with couple stresses. Steady flow of blood through modeled vascular stenosis was investigated by McDonald [6]. Shukla et al. [7] discussed the effect of stenosis on non-Newtonian flow of blood in an artery but later on they took into account the effect of radial distribution of cells and the existence of the peripheral plasma layer near the wall, and studied the flow of blood through an artery with mild stenosis by assuming blood as a power law fluid. Blood flow through an artery with mild stenosis was investigated by Sinha and Singh [8] and couple stresses effects on blood flow were also discussed by them. Srivastava [10] studied the flow through stenotic blood vessel by considering blood as a couple stress fluid. Lee [11] developed a numerical study on flow of fluid through tubes with double constrictions. Misra et al. [14] presented a non-Newtonian model in which they discussed flow of blood through arteries under stenotic conditions. Haldar and Ghosh [15] studied blood flow under the effect of a magnetic field through an indented artery in the presence of erythrocytes taking blood as a Newtonian fluid and discussed the expressions for blood velocity, pressure and flow rate. Murata [16] proposed a sedimentation model on flow properties of aggregating red cell suspension in constricted horizontal tubes and assumed a constant hematocrit level and the Newtonian viscosity in the central region of circular tubes. Chakravarty and Mandal [17] investigated a two dimensional flow of blood under stenotic conditions through tapered artery. Srivastava [18] analysed the flow of blood in a mild stenosed artery taking the central layer (core region) as a couple stress fluid as it is the suspension of erythrocytes (RBC) and a peripheral layer of plasma as a Newtonian fluid. Pralhad and Schultz [19] studied the modeling of arterial stenosis and employed its application to blood diseases taking blood as a non- Newtonian couple stress fluid.

A large number of investigations have been presented by several researchers to understand peristalsis through a permeable medium under the influence of body acceleration by considering blood as a Newtonian or non-Newtonian fluid. When our body experiences a sudden change in velocity, blood flow is disturbed. Although human body has remarkable adaptation to such type of sudden change, even then a prolonged exposure of body to such vibrations may lead to many health problems. While driving vehicles (car, bus, motorcycle, etc.), travelling in aircraft, spacecraft, jogging, etc., the human body is quite subjected to accelerations or vibrations, and they may be the reason of many health issues like increasing pulse rate, headache, losing vision, and abdominal pain. Rathod and Tanveer [20] discussed the effects of periodic body acceleration on pulsatile blood flow through a porous medium under the influence of a magnetic field and reported that local exposure to a magnetic field could relax blood vessel and enhance blood flow. Varshney et al. [21] also presented the effect of a magnetic field on blood flow in a multiple stenosed artery and reported that under a strong magnetic field effect, the flow rate of the streaming blood reduces up to $40 \%$.

Shit and Roy [22] proposed a theoretical study of hydro- magnetic pulsatory blood flow in a channel which is constricted and porous and provided evidence for the vital role of hydro-dynamic factors in the development and the progression of stenosis in an artery. Rathee and Singh [23] employed an analysis of a two- layered model of blood flow under the effect of a magnetic field through a stenosed artery in a porous medium and concluded that as effects of the magnetic field increase, the velocity profile of the blood flow under given conditions shows a reverse behavior. Eldesoky [24] studied an unsteady MHD pulsatile blood flow through a stenotic channel in a porous medium with slip at permeable walls subjected to time dependent velocity (injection/ suction). Siddiqui and Geeta [25] investigated a mathematical model for blood flow through inclined stenosed artery and presented the influence of the inclination angle. The unsteady slip flow of blood through constricted artery was investigated by Gaur and Gupta [26]. They reported the variation in flow characteristics along the axial distance with passage of time. Elangovan and Selvaraj [27] analysed the blood flow through a multiple stenosed artery with periodic body acceleration in the presence of a magnetic field. Malek and Horque [28] recently presented a theoretical study on the flow of blood through a stenosed artery with permeable wall taking into the consideration of the hematocrit level. Sankad and Nagathan [29] 
discussed the transport of an MHD couple stress fluid through peristalsis in a porous medium under the influence of the heat transfer and slip effects. Tripathi and Sharma [30] focused on the study of effects of heat and mass transfer on an arterial blood (with variable viscosity) flow in the presence of the applied magnetic field with chemical reaction.

Motivated by the above investigations and in order to simulate the constricted artery flow problem, we have made an attempt to present a theoretical analysis to explore the significant influence of slip velocity and body acceleration on the flow of non-Newtonian blood through a stenosed artery with permeable wall in the presence of an externally applied magnetic field. Since selective permeability of the arterial wall permits applying slip condition in place of no-slip condition, it is another close step to the real situation of flow of blood at wall of a narrow artery as the arterial permeable walls allow the fluid particles to slip at boundary. Extensive quantitative analysis is set up by applying the Laplace and finite Hankel transformations to estimate quantitative effects of slip velocity, body acceleration, and applied magnetic field on flow of blood through an artery so as to substantiate the utility of the present study.

\section{Formulation of problem}

In this study, we assume one dimensional motion of blood in a straight and rigid cylindrical stenosed artery through a porous medium by considering blood as a non-Newtonian elastico-viscous, incompressible and electrically conducting fluid under the influence of a transversely applied magnetic field. It is well known that when a magnetic field is applied on an electrically conducting fluid like blood, an electromagnetic force will be generated. The electromotive force is given by the proportionality relation to the speed of motion and the magnetic field intensity by Ohm's law, which is given by

$$
\boldsymbol{J}=\sigma(\boldsymbol{E}+\boldsymbol{u} \times \boldsymbol{B})
$$

where $\boldsymbol{E}$ is the electric field intensity vector, $\sigma$ is the electrical conductivity, $\boldsymbol{u}$ is the velocity vector, $\boldsymbol{B}=\boldsymbol{B}_{0}+\boldsymbol{B}_{1}$ is the total magnetic flux intensity vector in which $\boldsymbol{B}_{1}$ is the negligibly small induced magnetic field vector) in comparison with the externally applied magnetic field $\boldsymbol{B}_{0}$ for a magneto-hydrodynamic (MHD) flow. We also assume that the electric field intensity vector $\boldsymbol{E}$ due to the polarization of charge is also negligible. Now the electromagnetic force is defined and included in the momentum equation as

$$
\boldsymbol{F}=\boldsymbol{J} \times \boldsymbol{B}=-\sigma B_{0}^{2} \boldsymbol{u} \quad \text { where } \quad\left|\boldsymbol{B}_{0}\right|=B_{0} .
$$

Further, we assume that the blood flow is laminar, unsteady, axially-symmetric and fully developed. We restrict our study to a one-dimensional blood flow along the axial direction of a cylindrical small artery.

Now the geometry of the arterial segment with symmetric shape of stenosis as proposed by Eldesoky [24] is

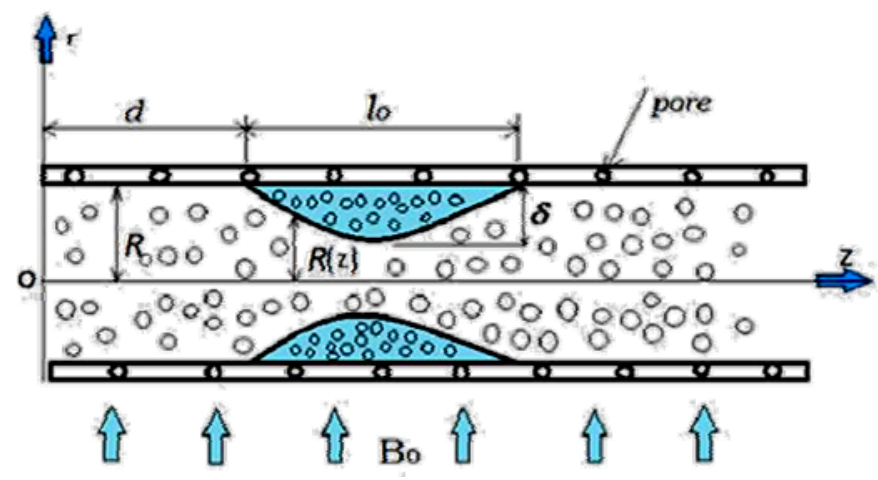

Fig.1. Geometrical representation of the arterial segment with stenosis. 


$$
\frac{R(z)}{R_{0}}=1-A\left[l_{0}^{s-1}(z-d)-(z-d)^{s}\right], \quad d \leq z \leq d+l_{0}
$$

where $\quad A=\frac{\epsilon}{R_{0} l_{0}^{s}} \frac{\frac{s}{s(s-1)}}{s-1}$,

and $s \geq 2$ is the stenosis shape parameter, $d$ is the position of stenosis, $l_{0}$ is the length of stenosis, $R(z)$ is the radius of stenosed vessel, $R_{0}$ is the radius of normal artery.

\section{Governing equations}

The Navier-Stokes equation for blood flow including the Lorenz force under the above assumptions, in cylindrical polar co-ordinates is

$$
\rho \frac{\partial u}{\partial t}=-\frac{\partial p}{\partial z}+\rho G(t)+\left(\mu+\mu_{1} \frac{\partial}{\partial t}\right) \nabla^{2} u-\frac{\mu}{k} u-\sigma B_{0}^{2} u
$$

where

$$
\begin{aligned}
& \nabla^{2} \cong\left(\frac{1}{r} \frac{\partial}{\partial r}\right)\left(r \frac{\partial}{\partial r}\right) . \\
& -\frac{\partial p}{\partial z}=p_{0}+p_{1} \cos \left(w_{p} t\right) ; \quad t \geq 0, \\
& G(t)=G_{0} \cos \left(w_{b} t+\phi\right) ; \quad t \geq 0
\end{aligned}
$$

where $p_{0}$ is the amplitude of the steady part of the pressure gradient, $p_{1}$ is the amplitude of the oscillatory pressure gradient, $w_{p}=2 \pi f_{p}$ with $f_{p}$ is the heart pulse frequency, $G_{0}$ represents the amplitude of body acceleration, $w_{b}=2 \pi f_{b}$ with $f_{b}$ as body acceleration frequency, $\varphi$ is the phase difference, $z$ is the axial direction. Then Eq.(2.5) is written as

$$
\rho \frac{\partial u}{\partial t}=p_{0}+p_{1} \cos \left(w_{p} t\right)+\rho G_{0} \cos \left(w_{b} t+\phi\right)+\left(\mu+\mu_{1} \frac{\partial}{\partial t}\right)\left(\frac{\partial^{2} u}{\partial r^{2}}+\frac{1}{r} \frac{\partial}{\partial r}\right)-\frac{\mu}{K} u-B_{0}^{2} u
$$

where
$u(r, t)$ is the component of velocity in the axial direction,
$\rho$ is the density of blood,
$\mu$ is the blood viscosity coefficient,
$\mu_{1}$ is the blood elastic-viscosity coefficient,
$\sigma$ is the electrical conductivity of blood,
$K$ is the porosity coefficient,
$r$ is the radial co-ordinate,
$t$ is the time variable.

Let us introduce the following non-dimensional quantities 


$$
\begin{aligned}
& u^{\prime}=\frac{u}{w_{p} R_{0}}, \quad r^{\prime}=\frac{r}{R_{0}}, \quad p_{0}^{\prime}=\frac{R_{0}}{w_{p} \mu} p_{0}, \quad p_{1}^{\prime}=\frac{R_{0}}{w_{p} \mu} p_{1}, \quad z^{\prime}=\frac{z}{R_{0}}, \quad t^{\prime}=t w_{p}, \\
& b=\frac{w_{b}}{w_{p}}, \quad G_{0}^{\prime}=\frac{\rho R_{0}}{\mu w_{p}} G_{0}, \quad K^{\prime}=\frac{K}{R_{0}^{2}} .
\end{aligned}
$$
Eq.(3.5) as

After applying non-dimensional terms and on dropping primes, we have a simplified form of

$$
\alpha^{2} \frac{\partial u}{\partial t}=p_{0}+p_{1} \cos t+G_{0} \cos (b t+\phi)+\left(1+\beta \frac{\partial}{\partial t}\right)\left(\frac{\partial^{2} u}{\partial r^{2}}+\frac{1}{r} \frac{\partial u}{\partial r}\right)-\left(H^{2}+M^{2}\right) u
$$

where $\alpha=R_{0} \sqrt{\frac{\rho \omega}{\mu}}$ is the Womersley parameter, $\mathrm{H}=B_{0} R_{0} \sqrt{\frac{\sigma}{\mu}}$ is the Hartmann number, $M=\sqrt{\frac{1}{K}}$ is the permeability parameter and $\beta=\frac{\mu_{1} \omega}{\mu}$.

Further, we assume that at $t<0$, only heart pumping action is present and at $t=0$, the blood streams in the artery due to the instantaneous pressure gradient which is given by

$$
-\frac{\partial p}{\partial z}=p_{0}+p_{1}
$$

\section{The initial and boundary conditions}

When intensity of permeability is small, the boundary condition proposed by Beaver and Joseph [1] is applicable which was further simplified by Saffman [4] (also known as Saffman's Slip condition) as $\frac{d u}{d r}=\frac{\eta}{\sqrt{K}} u$ where $\eta$ is constant depending on the porous material properties and on its structure, $K$ is the permeability parameter (or Darcy number) of the porous material of the wall. This condition is also applicable to an unsteady flow as well as to MHD fluid flows. Now the initial and boundary conditions are prescribed as follows

$$
\frac{\partial u}{\partial r}=-h u
$$

or

$u^{\prime}=-h u$,

or

$$
u^{\prime}+h u=0 \quad \text { when } \quad r=a \text { and } \quad t \geq 0
$$

where

$$
h=-\frac{\eta}{R_{0} \sqrt{K}} \quad \text { and } \quad a=\frac{R(z)}{R_{0}},
$$

$$
u(r, 0)=\sum_{n=1}^{\infty} \frac{2 h}{a} \frac{p_{0}+p_{1}}{\left(H^{2}+M^{2}+\lambda_{n}^{2}\right)\left(\lambda_{n}^{2}+h^{2}\right)} \frac{J_{0}\left(r \lambda_{n}\right)}{J_{l}\left(a \lambda_{n}\right)} \quad \text { for } \quad 0 \leq r \leq a,
$$

$$
u(0, t) \text { is finite. }
$$




\section{Required integral transformations}

If $g(t)$ is a continuous function of $t$ and is of exponential order for $t \geq 0$, then its Laplace transformation is defined as

$$
\overline{g(s)}=\int_{0}^{\infty} e^{-s t} g(t) d(t), \quad s>0,
$$

and if $f(t)$ satisfies the Dirichlet condition in a finite interval $[0, a]$ then its finite Hankel tranformation is defined as

$$
f\left(\lambda_{n}\right)=\int_{0}^{a} r f(r) J_{0}\left(r \lambda_{n}\right) d r
$$

where $\lambda_{n}$ are the roots of the equation $\lambda J_{0}^{\prime}+h J_{0}=0$, where $J_{0}(r)$ and $J_{l}(r)$ are Bessel functions of the first kind. Then $f(r)$ is given by

$$
f(r)=\frac{2}{a^{2}} \sum_{n=1}^{\infty} \frac{\lambda_{n}^{2}}{\left(h^{2}+\lambda_{n}^{2}\right)} \frac{J_{0}\left(r \lambda_{n}\right)}{J_{0}^{2}\left(a \lambda_{n}\right)} f\left(\lambda_{n}\right) .
$$

\section{Analysis}

Applying the Laplace transformation to Eq.(3.7) in the light of Eq.(5.1), we have

$$
\begin{aligned}
& \alpha^{2}(s \overline{u(r, s)}-u(r, 0))=p_{0} \frac{1}{s}+p_{1}\left(\frac{s}{1+s^{2}}\right)+G_{0}\left(\frac{\mathrm{s} \cos \varphi-\mathrm{b} \sin \varphi}{\mathrm{s}^{2}+b^{2}}\right)+ \\
& +\left(\frac{\delta^{2}}{\delta r^{2}}+\frac{1}{r} \frac{\delta}{\delta r}\right)(\overline{u(r, s)}+\beta s \overline{u(r, s)}-u(r, 0))-\left(H^{2}+M^{2}\right) \overline{u(r, s)} .
\end{aligned}
$$

Applying the Hankel transformation to Eq.(6.1) in the light of Eq.(5.2), we obtain

$$
\begin{aligned}
& \left(\alpha^{2} s+H^{2}+M^{2}+\lambda_{n}^{2}+\beta s \lambda_{n}^{2}\right) \overline{u\left(\lambda_{n}, s\right)}+\left(\beta \lambda_{n}^{2}+\alpha^{2}\right) u\left(\lambda_{n}, 0\right)+ \\
& =\left(p_{0} \frac{1}{s}++G_{0}\left(\frac{s \cos \phi-b \sin \phi}{s^{2}+b^{2}}\right)\right) \frac{a}{\lambda_{n}} J_{l}\left(a \lambda_{n}\right),
\end{aligned}
$$

or

$$
\begin{aligned}
& \overline{u\left(\lambda_{n}, s\right)}=\left[\frac{1}{s+l}\left(\frac{p_{0}+p_{1}+G_{0} \cos \phi}{H^{2}+M^{2}+\lambda_{n}^{2}}\right) \frac{p_{0}}{\alpha^{2}+\beta \lambda_{n}^{2}}\left(\frac{1}{s(s+l)}\right)+\frac{p_{1}}{\alpha^{2}+\beta \lambda_{n}^{2}}\left(\frac{s}{\left(1+s^{2}\right)(s+l)}\right)+\right. \\
& \left.+\frac{G_{0} \cos \phi}{\alpha^{2}+\beta \lambda_{n}^{2}}\left(\frac{s}{\left(s^{2}+b^{2}\right)(s+l)}\right)-\frac{G_{0} b \sin \phi}{\alpha^{2}+\beta \lambda_{n}^{2}}\left(\frac{1}{\left(s^{2}+b^{2}\right)(s+l)}\right)\right] \frac{a}{\lambda_{n}} J_{l}\left(a \lambda_{n}\right)
\end{aligned}
$$

where

$$
l=\frac{H^{2}+M^{2}+\lambda_{n}^{2}}{\alpha^{2}+\lambda_{n}^{2}} .
$$


To obtain the expression for fluid velocity, applying the inverse Laplace transformation and Hankel transformation to Eq.(6.3), we get

$$
\begin{aligned}
& u(r, t)=\frac{2 h}{a} \sum_{n=1}^{\infty} \frac{J_{0}\left(r \lambda_{n}\right)}{\left(\lambda_{n}^{2}+h^{2}\right) J_{0}\left(a \lambda_{n}\right)}\left[\frac{p_{0}}{H^{2}+M^{2}+\lambda_{n}^{2}}+\right. \\
& +e^{-l t}\left(\frac{p_{0}+p_{1}+G_{0} \cos \phi}{H^{2}+M^{2}+\lambda_{n}^{2}}-\frac{l p_{1}}{\left(1+l^{2}\right)\left(\alpha^{2}+\beta \lambda_{n}^{2}\right)}-\frac{p_{0}}{l\left(H^{2}+M^{2}+\lambda_{n}^{2}\right)}\right)+ \\
& -\frac{G_{0}}{\left(l^{2}+b^{2}\right)\left(\alpha^{2}+\beta \lambda_{n}^{2}\right)}(l \cos \phi+b \sin \phi)+\frac{p_{1}}{\left(1+l^{2}\right)\left(\alpha^{2}+\beta \lambda_{n}^{2}\right)}(l \cos t+b \sin t)+ \\
& +\frac{G_{0}}{\left(l^{2}+b^{2}\right)\left(\alpha^{2}+\beta \lambda_{n}^{2}\right)}(l \cos (b t+\phi)+b \sin (b t+\phi)) .
\end{aligned}
$$

The expression for the fluid acceleration is given by

$$
F(r, t)=\frac{\partial u}{\partial t}
$$

or

$$
\begin{aligned}
& F(r, t)=\frac{2 h}{a} \sum_{n=1}^{\infty} \frac{J_{0}\left(r \lambda_{n}\right)}{\left(\lambda_{n}^{2}+h^{2}\right) J_{0}\left(a \lambda_{n}\right)}\left[-l e^{-l t}\left(\frac{A_{0}+p_{1}+G_{0} \cos \phi}{H^{2}+M^{2}+\lambda_{n}^{2}}+\right.\right. \\
& \left.-\frac{l p_{1}}{\left(1+l^{2}\right)\left(\alpha^{2}+\beta \lambda_{n}^{2}\right)}-\frac{p_{0}}{l\left(H^{2}+M^{2}+\lambda_{n}^{2}\right)}\right)-\frac{G_{0}}{\left(l^{2}+b^{2}\right)\left(\alpha^{2}+\beta \lambda_{n}^{2}\right)}(l \cos \phi+b \sin \phi)+ \\
& \left.+\frac{p_{1}}{\left(1+l^{2}\right)\left(\alpha^{2}+\beta \lambda_{n}^{2}\right)}(-l \sin t+\cos t)+\frac{G_{0}}{\left(l^{2}+b^{2}\right)\left(\alpha^{2}+\beta \lambda_{n}^{2}\right)}\left(-l b \sin (b t+\phi)+b^{2} \cos (b t+\phi)\right)\right] .
\end{aligned}
$$

Similarly, we can obtain the expression for the volumetric flow rate, which is given by

$$
Q(z, t)=2 \pi \int_{0}^{a} r u(r, t) d r,
$$

or

$$
\begin{aligned}
& Q(z, t)=\frac{4 \pi h}{a} \sum_{n=1}^{\infty} \frac{J_{l}\left(a \lambda_{n}\right)}{\lambda_{n}\left(\lambda_{n}^{2}+h^{2}\right)}\left[\frac{p_{0}}{H^{2}+M^{2}+\lambda_{n}^{2}}+e^{-l t}\left(\frac{p_{0}+p_{1}+G_{0} \cos \phi}{H^{2}+M^{2}+\lambda_{n}^{2}}+\right.\right. \\
& \left.-\frac{l p_{1}}{\left(1+l^{2}\right)\left(\alpha^{2}+\beta \lambda_{n}^{2}\right)}-\frac{p_{0}}{l\left(H^{2}+M^{2}+\lambda_{n}^{2}\right)}\right)-\frac{G_{0}}{\left(l^{2}+b^{2}\right)\left(\alpha^{2}+\beta \lambda_{n}^{2}\right)}(l \cos \phi+b \sin \phi)+ \\
& \left.+\frac{p_{1}}{\left(1+l^{2}\right)\left(\alpha^{2}+\beta \lambda_{n}^{2}\right)}(l \cos t+b \sin t)+\frac{G_{0}}{\left(l^{2}+b^{2}\right)\left(\alpha^{2}+\beta \lambda_{n}^{2}\right)}(l \cos (b t+\phi)+b \sin (b t+\phi))\right] .
\end{aligned}
$$

Also, the expression for the shear stress is calculated as 


$$
\tau(r, t)=\mu \frac{\partial u}{\partial r}
$$

or

$$
\begin{aligned}
& \tau(r, t)=\frac{2 \mu h}{a} \sum_{n=1}^{\infty} \frac{\left(-\lambda_{n}\right) J_{l}\left(r \lambda_{n}\right)}{\left(\lambda_{n}^{2}+h^{2}\right) J_{0}\left(a \lambda_{n}\right)}\left[\frac{p_{0}}{H^{2}+M^{2}+\lambda_{n}^{2}}+e^{-l t}\left(\frac{p_{0}+A_{1}+G_{0} \cos \phi}{H^{2}+M^{2}+\lambda_{n}^{2}}+\right.\right. \\
& \left.\left.-\frac{l p_{1}}{\left(1+l^{2}\right)\left(\alpha^{2}+\beta \lambda_{n}^{2}\right)}-\frac{p_{0}}{l\left(H^{2}+M^{2}+\lambda_{n}^{2}\right)}\right)-\frac{G_{0}}{\left(l^{2}+b^{2}\right)\left(\alpha^{2}+\beta \lambda_{n}^{2}\right)}(l \cos \phi+b \sin \phi)\right)+ \\
& +\frac{p_{1}}{\left(1+l^{2}\right)\left(\alpha^{2}+\beta \lambda_{n}^{2}\right)}(l \cos t+b \sin t)+\frac{G_{0}}{\left(l^{2}+b^{2}\right)\left(\alpha^{2}+\beta \lambda_{n}^{2}\right)}(l \cos (b t+\phi)+b \sin (b t+\phi)] .
\end{aligned}
$$

\section{Graphical results and discussion}

In the analysis of salient features of flow characteristics of blood e.g. the axial velocity, flow rate, wall shear stress and fluid acceleration, we obtained the expressions given in Eqs (6.4), (6.6), (6.8), (6.10) and exhibited through Figs 2-13. The effects of physical parameters defining fluid behaviour and flow geometries on the flow characteristics are discussed for different values of the Hartmann number $\mathrm{H}$, slip parameter $h$, amplitude of the body acceleration $G_{0}$, permeability parameter of the porous medium $M$ and are shown in Figs 2 to 13. Figure 2 shows that high magnetic field strength decreases the blood velocity which is in accordance with [22, 24]. It indicates that velocity of blood can be controlled by applying a suitable strength of the magnetic field. Figure 3 reveals through parabolic axial velocity profile that axial velocity has its maximum value at the centerline of the artery and a slip at wall fairly enhances? The blood flow velocity as compared to no-slip condition for different values of the slip parameter $h$, which is in agreement with the studies carried out by in the works [20, 22, 27, 30]. Figure 4 demonstrates the influence of amplitude of the body acceleration $G_{0}$ on axial velocity in the stenosed section and it is observed that by increasing slowly the value of the parameter $G_{0}$, we can increase fluid velocity but higher values show the reverse behaviour. Figure 5 shows that fluid velocity increases with increasing the permeability parameter of the porous medium and Womersley parameter whereas it decreases with increasing the Hartmann number and frequency of body acceleration. The volumetric flow rate exhibits approximately the same profile as the blood velocity which is shown in Figs 10 to 13.

It is widely accepted that the shear stress plays an important role in the growth of stenosis. So it is important to study the effects of flow parameters on it. Figure 6 shows the variation in the wall shear stress and it is observed that the wall shear stress decreases with increasing the magnetic parameter as reported in [24]. Figure 7 shows that wall shear stress decreases maximum at the throat of stenosis by increasing the values of the slip parameter $h$ as noticed in [24, 25, 26, 29]. Figure 8 shows that the effective viscosity and wall shear stress slightly decrease with body acceleration. Figure 9 shows that wall shear stress can be controlled by applying a suitable combination of values of the Hartmann number, slip parameter, amplitude of body acceleration and Womersley parameter. Finally, the fluid acceleration increases with an increase in the slip parameter, whereas decreases the magnitude of the wall shear stress. In contrast, flow acceleration caused by slippage at the stenotic throat reduces the wall shear stress but increases the flow rate with increasing the permeability parameter of the porous medium, Womersley parameter and slip parameter, whereas it decreases with increasing the Hartmann number and the frequency of body acceleration. It is also noticed that the effective viscosity and wall shear stress decrease with body acceleration but velocity and flow rate increase due to the wall slip velocity. 


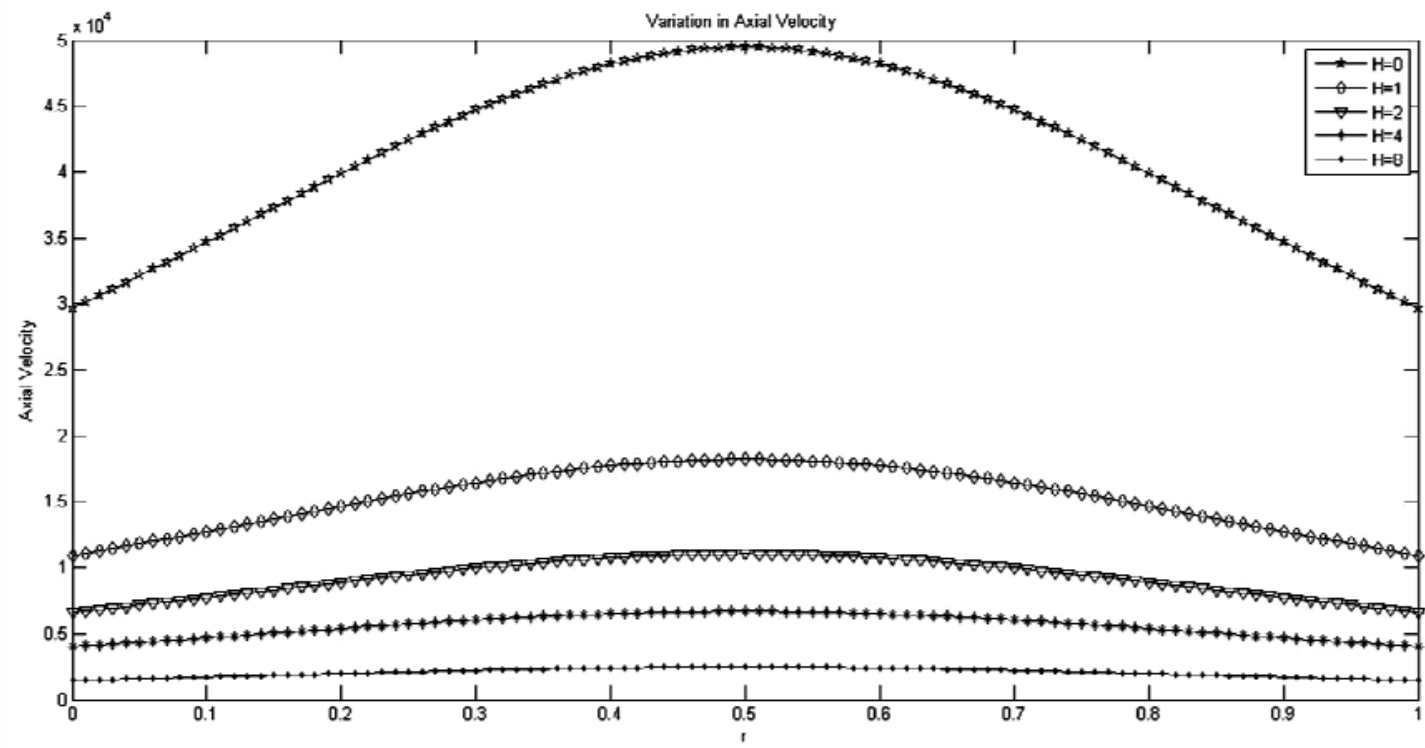

Fig.2. Variation in axial velocity; $b=2 ; G_{0}=3 ; p_{0}=2 ; p_{1}=4 ; t=1 ; \varphi=0.25 ; h=0.4 ; K=0.4$.

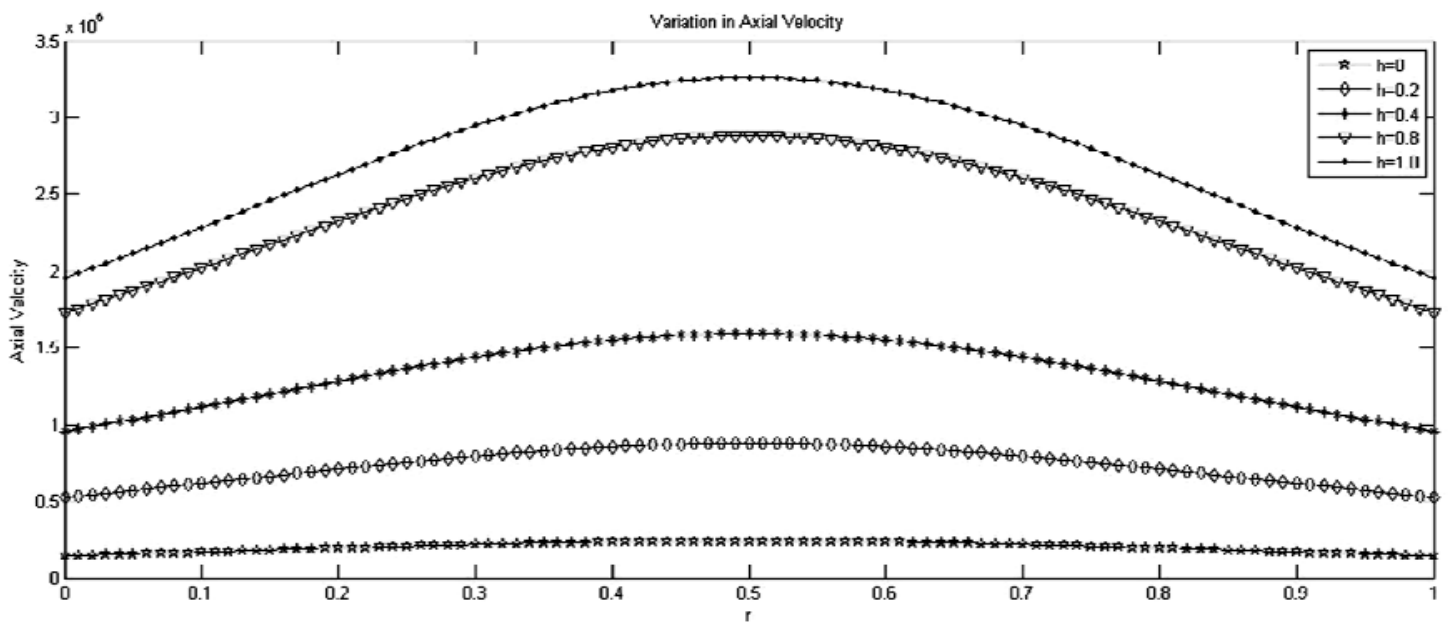

Fig.3. Variation in axial velocity; $b=2 ; G_{0}=3 ; p_{0}=2 ; p_{1}=4 ; t=1 ; \varphi=0.25 ; \mathrm{H}=2.5 ; K=0.4$.

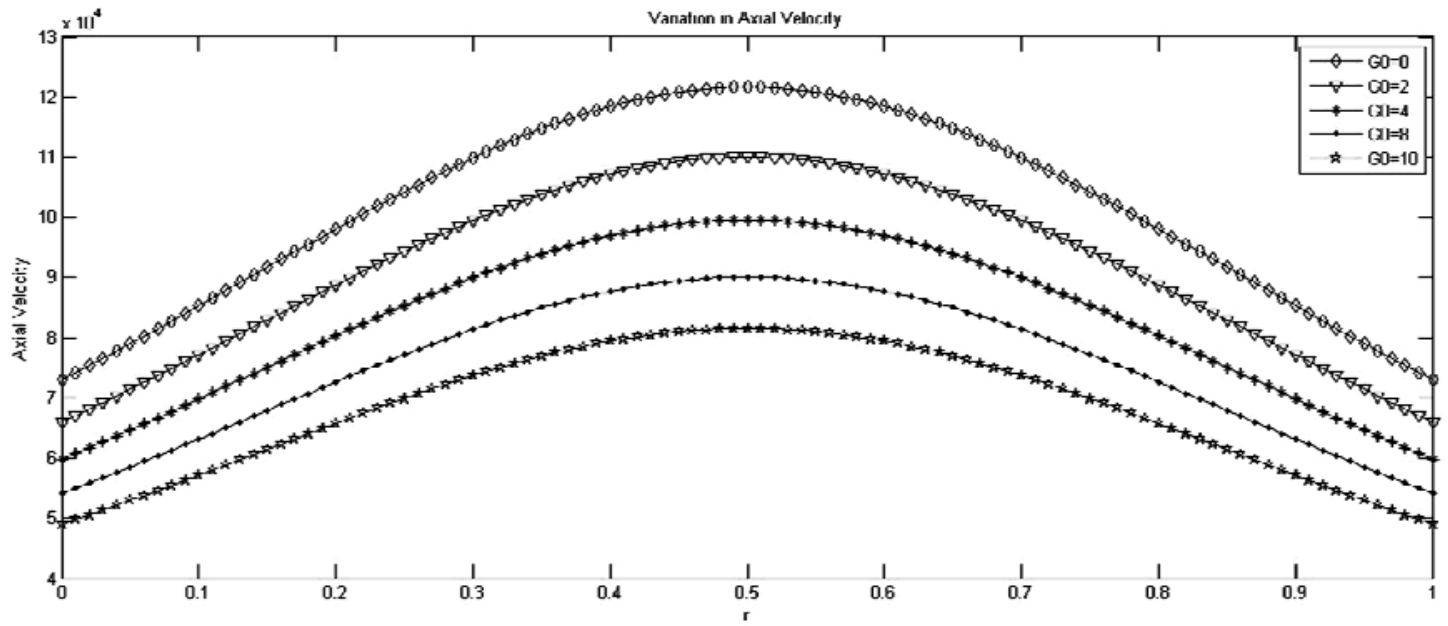

Fig.4. Variation in axial velocity; $b=2 ; \mathrm{H}=2.5 ; p_{0}=2 ; p_{1}=4 ; t=1 ; \varphi=0.75 ; h=0.4 ; K=0.4$. 


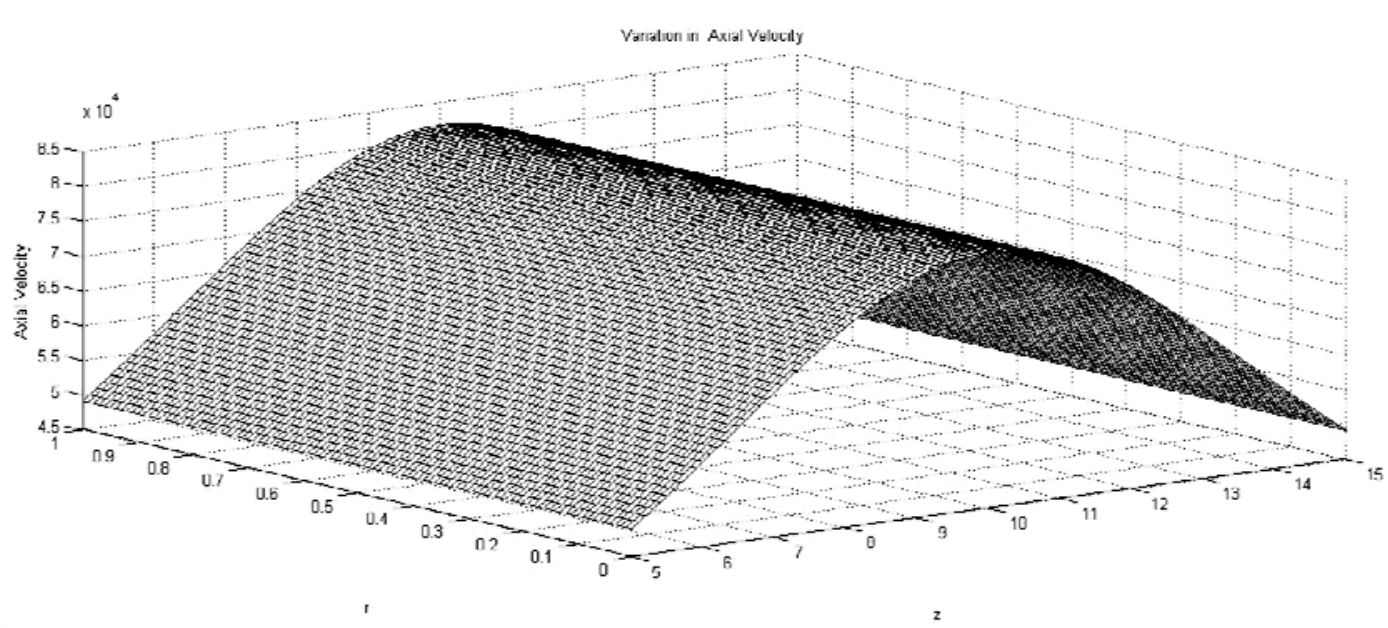

Fig.5. Variation in axial velocity; $b=2 ; G_{0}=3 ; p_{0}=2 ; \varphi=0.50 ; \mathrm{H}=4 ; p_{1}=4 ; t=1 ; h=0.4 ; K=0.4$.

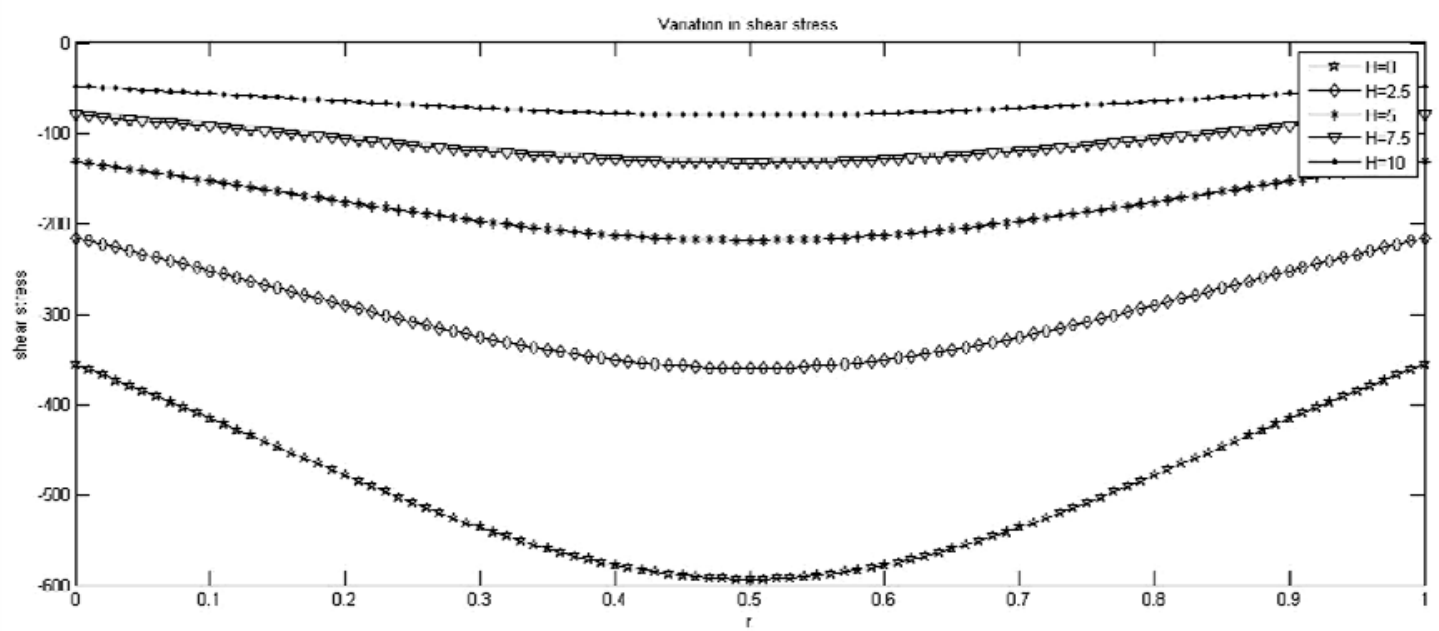

Fig.6. Variation in wall shear stress; $b=2 ; G_{0}=3 ; p_{0}=2 ; p_{1}=4 ; t=1 ; \varphi=0.60 ; h=0.4 ; K=0.4$.

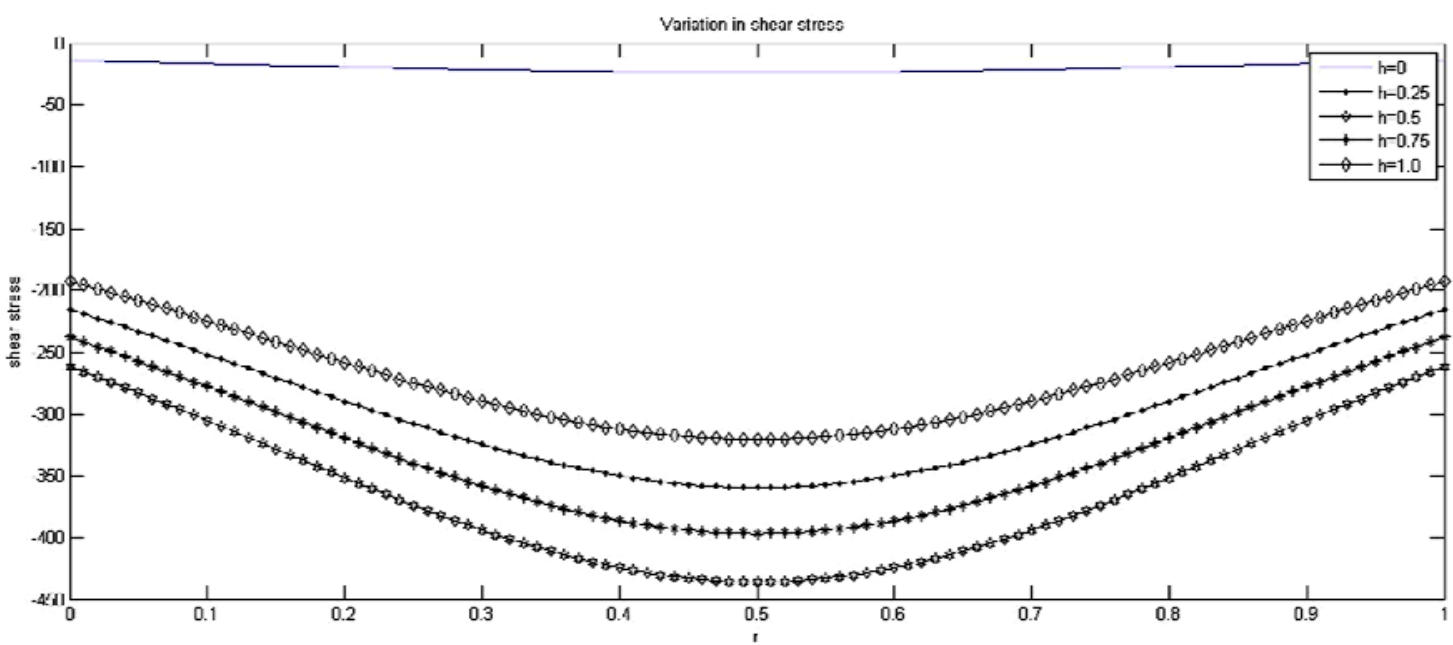

Fig.7. Variation in wall shear stress; $b=2 ; G_{0}=3 ; p_{0}=2 ; p_{1}=4 ; t=1 ; \varphi=0.60 ; \mathrm{H}=4 ; K=0.4$. 


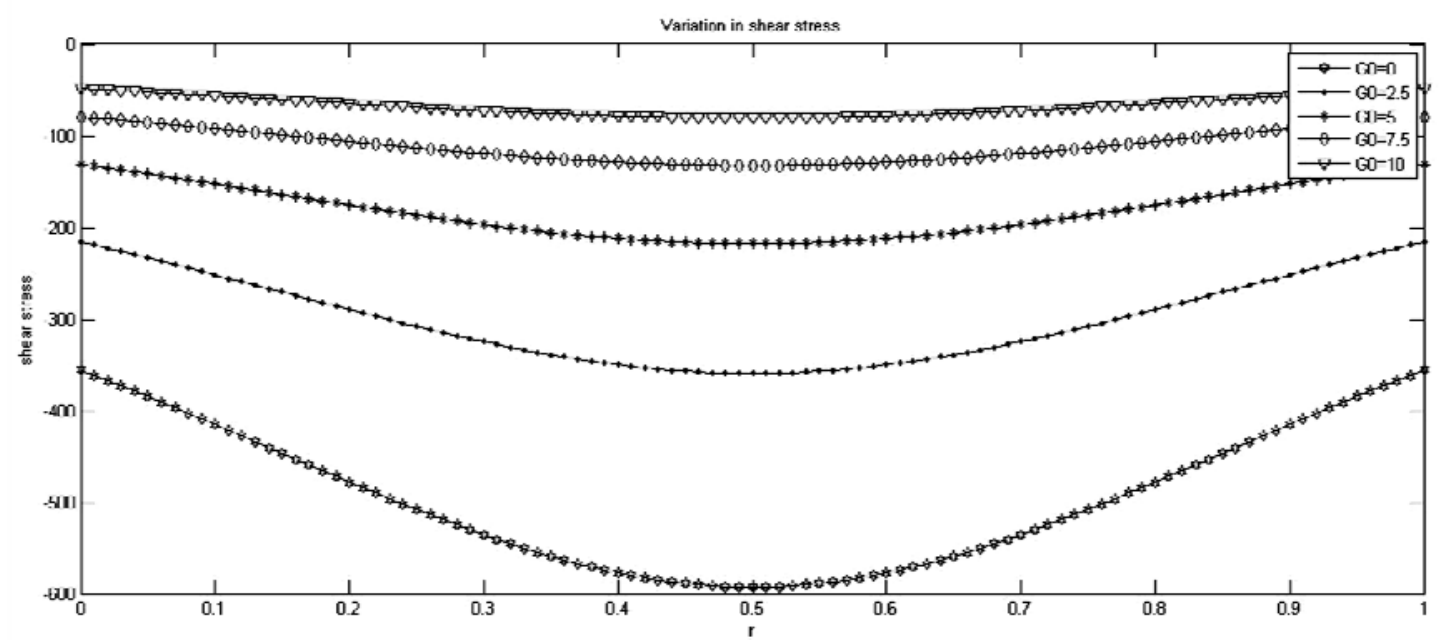

Fig.8. Variation in wall shear stress; $b=2 ; \mathrm{H}=2.5 ; p_{0}=2 ; p_{1}=4 ; t=1 ; \varphi=0.75 ; h=0.4 ; K=0.4$.

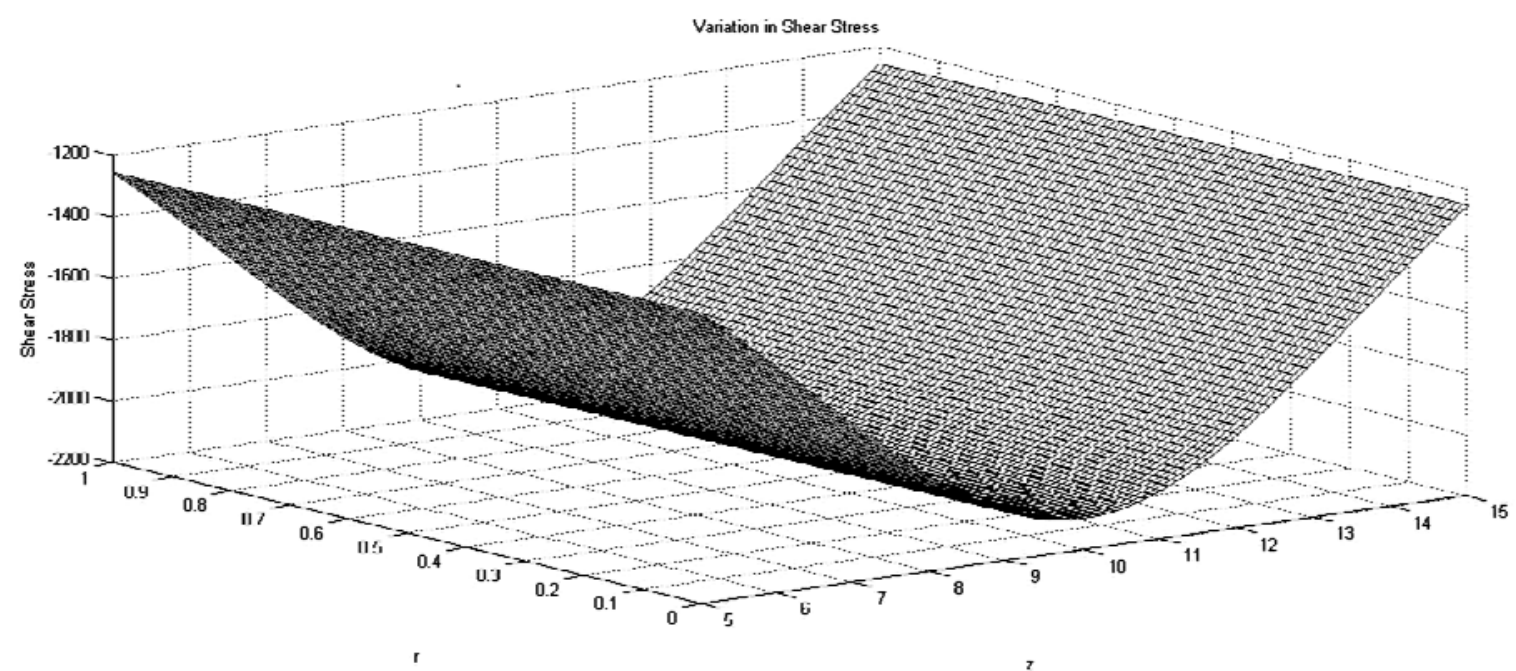

Fig.9. Variation in wall shear stress; $b=2 ; G_{0}=3 ; p_{0}=2 ; p_{1}=4 ; t=1 ; \varphi=0.75 ; h=0.4 ; K=0.4 ; \mathrm{H}=5$.

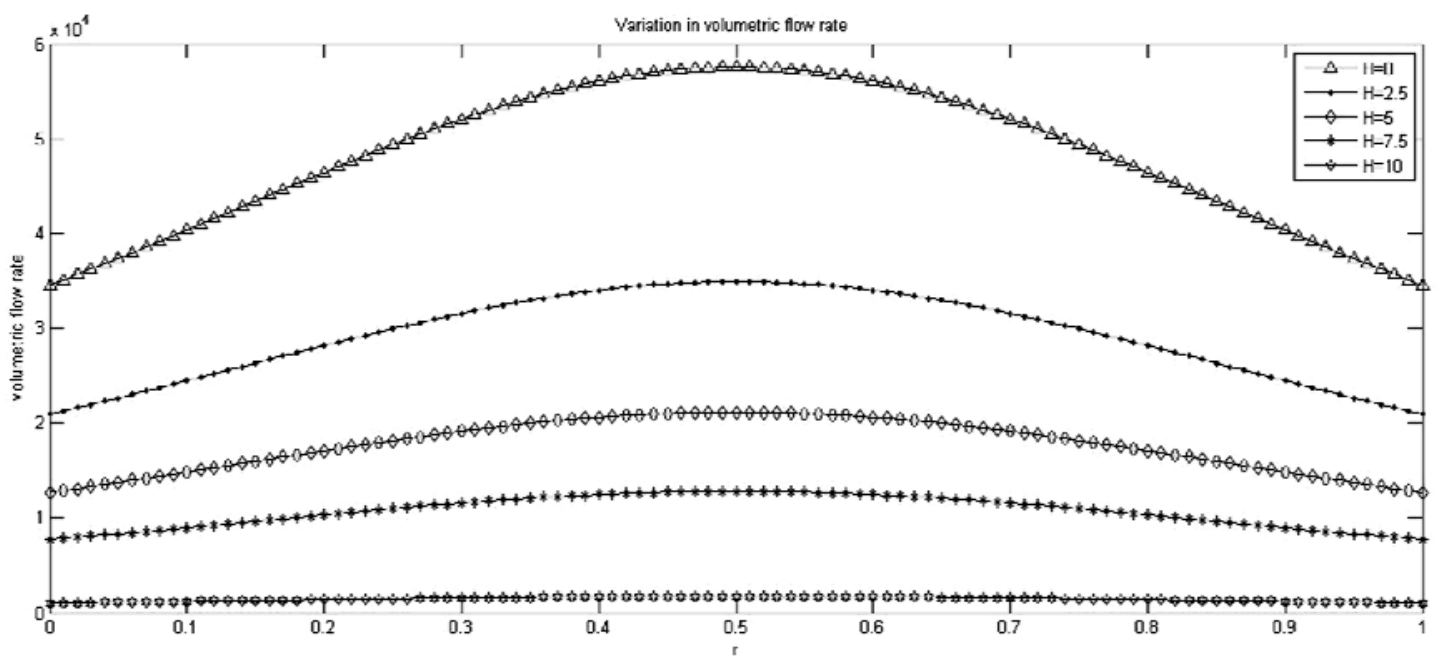

Fig.10. Variation in volumetric flow rate; $b=2 ; p_{0}=2 ; p_{1}=4 ; h=0.4 ; t=1 ; \phi=0.60 ; \mathrm{H}=2.5 ; K=0.4$. 


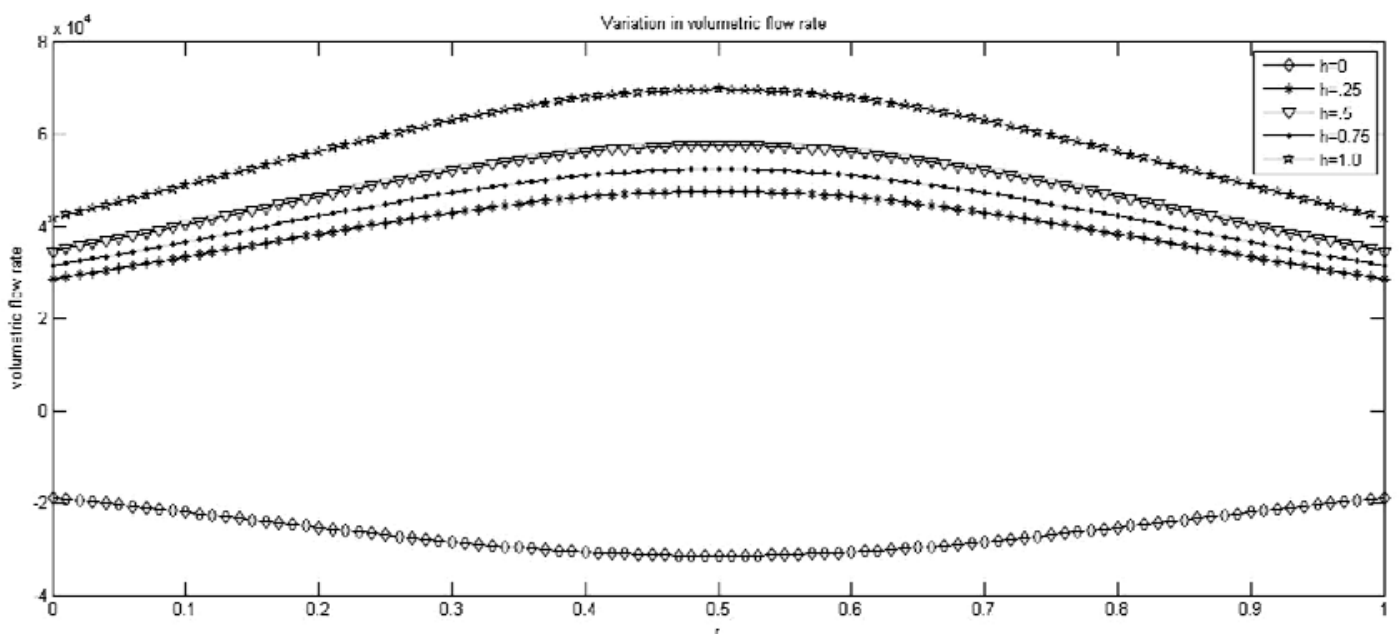

Fig.11. Variation in volumetric flow rate; $b=2 ; G_{0}=3 ; p_{0}=2 ; p_{1}=4 ; t=1 ; \phi=0.25 ; h=0.4 ; K=0.4$.

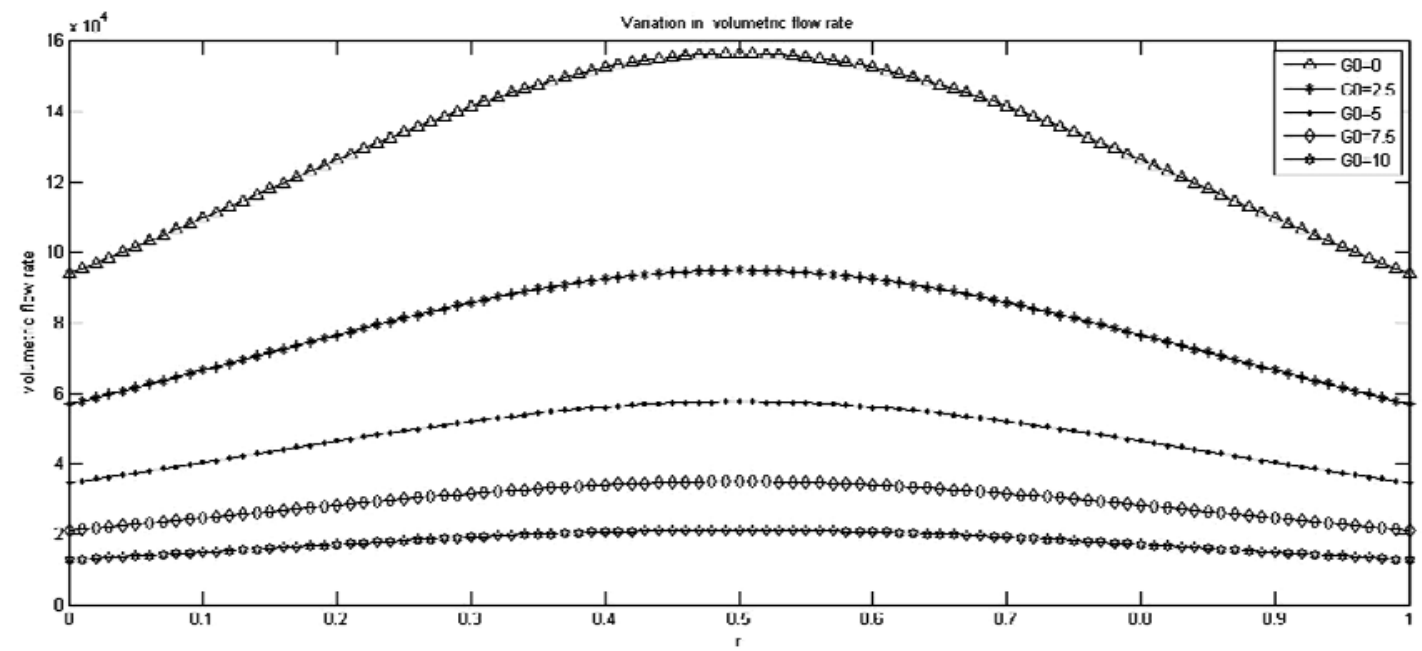

Fig.12. Variation in volumetric flow rate; $b=2 ; \mathrm{H}=2.5 ; p_{0}=2 ; p_{1}=4 ; t=2 ; \varphi=0.75 ; h=0.4 ; K=0.4$.

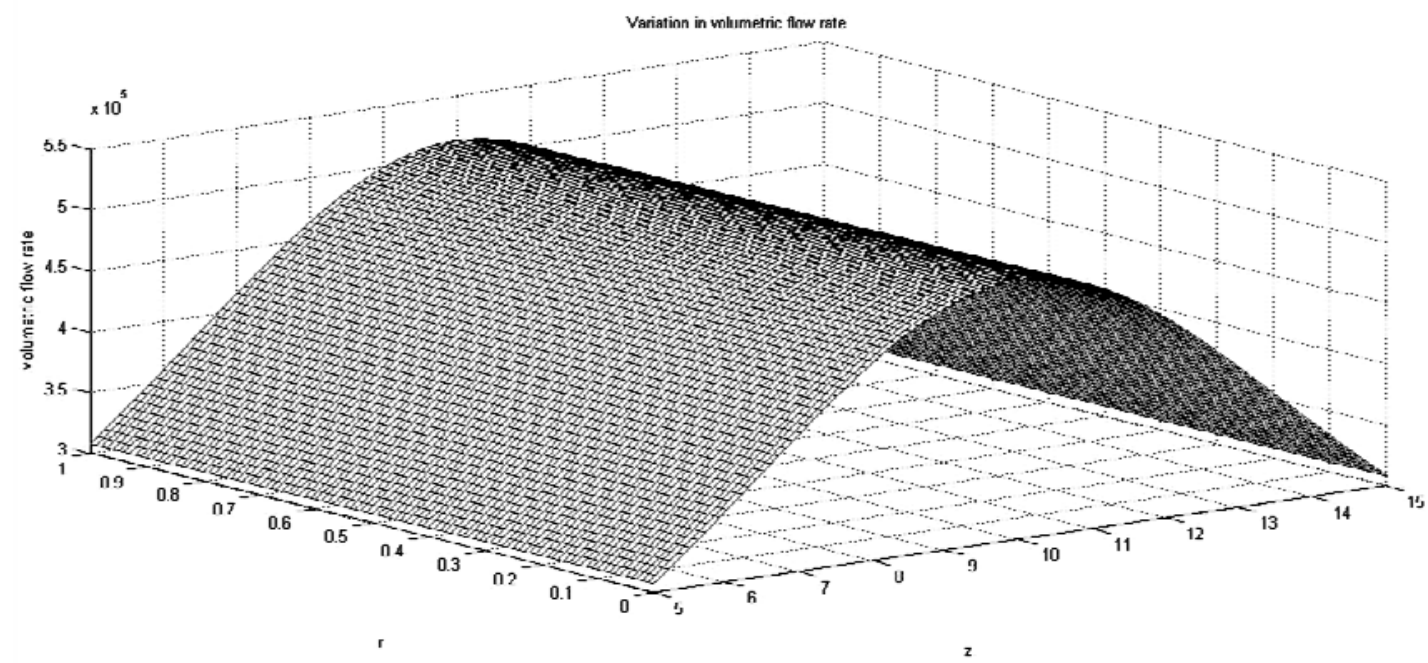

Fig.13. Variation in volumetric flow rate; $b=2 ; G_{0}=3 ; p_{0}=2 ; p_{1}=4 ; t=1 ; \varphi=0.75 ; h=0.4 ; K=0.4 ; \mathrm{H}=5$. 


\section{Conclusion}

In this investigation, effects of the wall slip and body acceleration on the blood flow through a constricted artery in a porous medium under the influence of the applied magnetic field have been analyzed. In this study, the assumption of the slip velocity at the permeable wall is of prime concern. Some effective results of the article are summarized below as:

(i) The pulsatile axial velocity and volumetric flow rate increases considerably with the increase of slip velocity and body acceleration parameters in the presence of the magnetic field and porosity.

(ii) There is a considerable decline in the wall shear stress at the throat of stenosis with an enhancement of slip velocity and body acceleration parameters in the presence of the magnetic field.

(iii) The application of the magnetic field is helpful in controlling the axial blood flow since high strength of the magnetic field causes a decrease in the axial speed of blood.

(iv) A significant growth in the velocity slip as well as the body acceleration leads to a considerable growth in the streaming blood velocity and volumetric flow rate with the applied magnetic field and porosity. This suggests that the blood flow through stenosed arteries can be enhanced by increasing the application of slip inducing drugs as well as the body acceleration.

(iv) When the blood flow rises, the heart has to pump blood at a faster rate. Hence cardiovascular disease patients should be advised to avoid strenuous activities in order to avoid the excessive pressure on the heart.

(v) Evidently the application of suitable slip inducing drugs and the magnetic field can control the wall shear stress within the stenosed section of the porous artery.

A proper understanding of the interaction of the body acceleration and slip velocity with the rheological properties of the streaming blood may contribute to the treatment of cardiovascular diseases. Such a combined investigation of the body acceleration and slip velocity can also be useful in the diagnosis and treatment of some health problems like vision loss, joint's pain and vascular problems. By applying an appropriate magnetic field, it is possible to control blood pressure and also it is effective for conditions such as poor circulation (nacrosis), headaches, joint's pain, travel sickness, muscle pain, etc. The slip condition plays a significant role in spurt, hysteresis effects and shear skin. The fluids with boundary slip have useful technological and clinical applications; for instance in polishing artificial heart valves and internal cavities.

\section{Nomenclature}

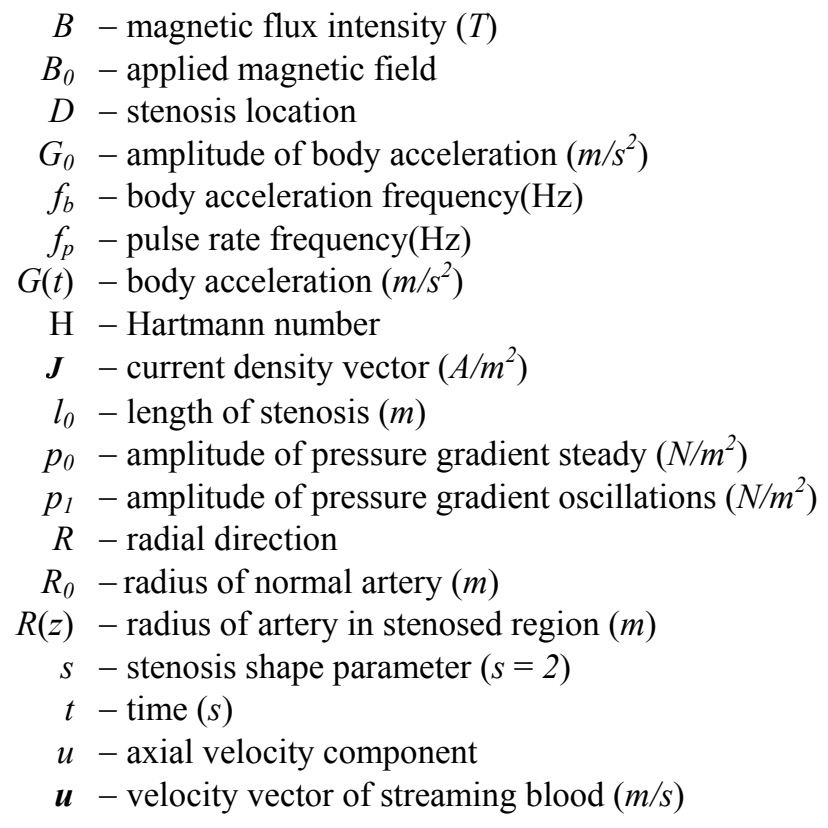


$\alpha$ - Womersley frequency parameter

$\mu \quad-$ dynamic viscosity of blood $(\mathrm{kg} / \mathrm{ms})$

$\mu_{l}-$ particle concentration parameter

$\rho$ - density of blood $\left(\mathrm{kg} / \mathrm{m}^{3}\right)$

$\sigma$ - electrical conductivity $(S / m)$

\section{References}

[1] Beaver G.S. and Joseph D.D. (1967): Boundary conditions at a naturally permeable wall. - J. Fluid Mech., vol.30, pp.197-207.

[2] Oka S. and Murata T. (1970): A theoretical study of flow of blood in a capillary with permeable wall. - Jpn. J. Appl. Sci., vol.9, pp.345-352.

[3] Saffman P.D. (1971): On the boundary conditions at the surface of a porous medium. - Stud. Appl. Math., vol.50, pp.93-101.

[4] Popel A.S., Regirer S.A. and Usick P.I. (1974): A continuum model of blood flow. - Biorheology, vol.11, pp.427437.

[5] Yuan S.W. (1976): Foundation of Fluid Mechanics. - New Delhi: Prentice Hall of India Pvt. Ltd..

[6] McDonald D.A. (1979): On steady blood flow through modelled vascular stenosis. - J. Biomech., vol.12, pp.1320.

[7] Shukla J.B., Parihar R.S. and Rao B.R.P. (1980): Effect of stenosis on non-Newtonian flow of blood in an artery. - Bull. Math. Bio., vol.42, pp.283-294.

[8] Sinha P. and Singh C. (1984): Effects of couple stresses on the blood flow through an artery with mild stenosis. Biorheology, vol.21, pp.303-315.

[9] Fung Y.C. (1984): Biodynamics - Circulation. - New York: Springer Verlag.

[10] Srivastava L.M. (1985): Flow of couple stress fluid through stenotic blood vessels. - J. Biomech., vol.1, pp.479485.

[11] Lee T.S. (1990): Numerical studies of fluid flow through tubes with double constrictions. - J. Numer. Methods Fluids, vol.11, pp.1113-1126.

[12] Fung Y.C. (1990): Biodynamics Motion, Flow, Stress and Growth. - New York: Springer Verlag.

[13] Mazumdar J.N. (1992): Biofluid Mechanics. - Singapore: World Scientific.

[14] Misra J.C., Patra M.K. and Misra S.C. (1993): A non-Newtonian model for blood flow through arteries under stenotic conditions. - J. Biomech., vol.26, pp.1129-1141.

[15] Haldar K. and Ghosh S.N. (1994): Effect of a magnetic field on blood flow through an indented tube in the presence of erythrocytes. - Indian J. Pure Appl. Math., vol.25, pp.345-352.

[16] Murata T. (1998): Theoretical analysis of flow properties of aggregating red cell suspensions in narrow horizontal tubes. - Clini. Hemorh., vol.14, pp.519-530.

[17] Chakravarty S. and Mandal P.K. (2001): Two-dimentional blood flow through tapered arteries under stenotic conditions. - Int. J. Non-Linear Mech., vol.36, pp.731-741.

[18] Srivastava V.P. (2003): Flow of a couple stress fluid representing blood through stenotic vessels with a peripheral layer. - Indian J. Pure Appl. Math., vol.34, pp.1727-1740.

[19] Pralhad R.N. and Schultz D.H. (2004): Modelling of arterial stenosis and its applications to blood diseases. - J. Math. Biosci., vol.190, pp.203-220. 
[20] Rathod V.P. and Tanveer S. (2009): Pulsatile flow of couple stress fluid through a porous medium with periodic body acceleration and magnetic field. - Bull. Malaysian Math. Sci. Soc., vol.32, pp.245-259.

[21] Varshney G., Katiyar V.K. and Kumar S. (2010): Effect of magnetic field on the blood flow in artery having multiple stenosis. - A numerical Study; Int. J. Eng. Sci. and Technol., vol.2, pp.67-82.

[22] Shit G.C. and Roy M. (2012): Hydro-magnetic pulsatory flow of blood in a constricted porous channel. - A Theoretical Study; Proc. World Congr. Eng., vol.1, pp.83-88.

[23] Rathee R. and Singh J. (2013): Analysis of two-layered model of blood flow through composite stenosed artery in porous medium under the effect of magnetic field. - J. Rajasthan Academy Phys. Sci., vol.12, pp.259-276.

[24] Eldesoky I.M.I. (2014): Unsteady MHD pulsatile blood flow through porous medium in stenotic channel with slip at permeable walls subjected to time dependent velocity (injection/ suction). - Walailak J. Sci. Tech., vol.11, No.11, pp.901-922.

[25] Siddiqui S.U., Shah S.R. and Geeta (2014): Effect of body acceleration and slip velocity on the pulsatile flow of casson fluid through stenosed artery. - Adv. Appl. Sci. Res., vol.5, No.3, pp.213-225.

[26] Gaur M. and Gupta M.K. (2015): Unsteady slip flow of blood through constricted artery. - Adv. Appl. Sci. Res., vol.6, pp.49-58.

[27] Elangovan K. and Selvaraj K. (2016): Study of multiple stenosed artery with periodic body acceleration in presence of magnetic field. - Int. J. Sci. Res. Manag., vol.4, No.06, pp.4214- 4226.

[28] Malek A. and Horque A. (2017): Hematocrit level on blood flow through a stenosed artery with permeable wall. - Int. J. Appl. Appl. Math., vol.12, No.1, pp.291-304.

[29] Sankad G.C. and Nagathan P.S. (2017): Transport of MHD couple stress fluid through peristalsis in a porous medium under the influence of heat transfer and slip effects. - Int. J. of Appl. Mech. Engg., vol.22, No.2, pp.403-414

[30] Tripathi B. and Sharma B.K. (2018): Effect of variable viscosity on MHD inclined arterial blood flow with chemical reaction. - Int. J. of Appl. Mech. Engg., vol.23, No.3, pp.767-785.

Received: October 20, 2018

Revised: June 2, 2019 